\title{
ADAPTIVE CONTROL AND SYNCHRONIZATION OF RÖSSLER PROTOTYPE-4 SYSTEM
}

\author{
Sundarapandian Vaidyanathan ${ }^{1}$ \\ ${ }^{1}$ Research and Development Centre, Vel Tech Dr. RR \& Dr. SR Technical University \\ Avadi, Chennai-600 062, Tamil Nadu, INDIA \\ sundarvtu@gmail.com
}

\begin{abstract}
This paper investigates the adaptive control and synchronization of Rössler prototype-4 system with unknown parameters. The Rössler prototype-4 system is a classical three-dimensional chaotic system studied by O.E. Rössler (1979). First, adaptive control laws are designed to stabilize the Rössler prototype-4 system to its unstable equilibrium at the origin based on the adaptive control theory and Lyapunov stability theory. Then adaptive control laws are derived to achieve global chaos synchronization of identical Rössler prototype-4 systems with unknown parameters. Numerical simulations are shown to validate and illustrate the effectiveness of the proposed adaptive control and synchronization schemes for the Rössler prototype-4 system.
\end{abstract}

\section{KEYWORDS}

Adaptive Control, Chaos, Stabilization, Synchronization, Rössler Prototype-4 System.

\section{INTRODUCTION}

Chaotic systems are nonlinear dynamical systems that are highly sensitive to initial conditions. The sensitive nature of chaotic systems is commonly called as the butterfly effect [1].

Chaos is an interesting nonlinear phenomenon and has been extensively and intensively studied in the last two decades [1-30]. Chaos theory has been applied in many scientific disciplines such as Mathematics, Computer Science, Microbiology, Biology, Ecology, Economics, Population Dynamics and Robotics.

Thus, the behaviour of a chaotic system is highly unpredictable even if the system mathematical description is deterministic. The first three-dimensional chaotic system was discovered by Lorenz in 1963 [2], when he was studying weather models. From then on, many Lorenz-like chaotic systems such as Rössler system [3], Chen system [4], Lü system [5] and Liu system [6] were reported and analyzed.

The control of chaotic systems is to design state feedback control laws that stabilizes the chaotic systems around the unstable equilibrium points. Active control technique is used when the system parameters are known and adaptive control technique is used when the system parameters are unknown [7-10].

Synchronization of chaotic systems is a phenomenon that may occur when two or more chaotic oscillators are coupled or when a chaotic oscillator drives another chaotic oscillator. Because of

DOI : 10.5121/ijait.2011.1502 
International Journal of Advanced Information Technology (IJAIT) Vol. 1, No.5, October 2011

the butterfly effect, which causes the exponential divergence of the trajectories of two identical chaotic systems started with nearly the same initial conditions, synchronizing two chaotic systems is seemingly a very challenging problem in the chaos literature [11-38].

In 1990, Pecora and Carroll [11] introduced a method to synchronize two identical chaotic systems and showed that it was possible for some chaotic systems to be completely synchronized. From then on, chaos synchronization has been widely explored in a variety of fields including physical systems [12], chemical systems [13], ecological systems [14], secure communications [15-17], etc.

In most of the chaos synchronization approaches, the master-slave or drive-response formalism has been used. If a particular chaotic system is called the master or drive system and another chaotic system is called the slave or response system, then the idea of synchronization is to use the output of the master system to control the slave system so that the output of the slave system tracks the output of the master system asymptotically.

Since the seminal work by Pecora and Carroll [11], a variety of impressive approaches have been proposed for the synchronization of chaotic systems such as the OGY method [18], active control method [19-24], adaptive control method [25-30], sampled-data feedback synchronization method [31], time-delay feedback method [32], backstepping method [33], sliding mode control method [34-38], etc.

In this paper, we discuss the adaptive control and synchronization of the Rössler prototype-4 system (Rössler, [39], 1979) with unknown parameters. In the first part of the paper, we devise a state feedback control scheme for stabilizing the uncertain Rössler prototype-4 system about its unstable equilibrium at the origin. The stability results for adaptive control and parameter estimation are established using Lyapunov stability theory. In the second part of the paper, we devise a state feedback control scheme for synchronizing two identical uncertain Rössler prototype- 4 systems. The stability results for adaptive synchronization and parameter estimation are established using Lyapunov stability theory.

This paper is organized as follows. In Section 2, we derive results for the adaptive control of Rössler prototype-4 system with unknown parameters. In Section 3, we derive results for the adaptive synchronization of Rössler prototype- 4 systems with unknown parameters. Section 4 contains a summary of the main results derived in this paper.

\section{Adaptive Control of Rössler Prototype-4 Chaotic System}

\subsection{Theoretical Results}

The Rössler prototype-4 system (Rössler, 1979) is one of the classical three-dimensional chaotic systems. The Rössler prototype-4 system dynamics is described by

$$
\begin{aligned}
& \dot{x}_{1}=-x_{2}-x_{3} \\
& \dot{x}_{2}=x_{1} \\
& \dot{x}_{3}=a\left(x_{2}-x_{2}^{2}\right)-b x_{3}
\end{aligned}
$$

where $x_{i},(i=1,2,3)$ are the state variables and $a, b$ are positive constants.

The Rössler prototype-4 system (1) is chaotic when the parameter values are taken as

$$
a=0.5 \text { and } b=0.5
$$


The state orbits of the Rössler prototype-4 system (1) is described in Figure 1.

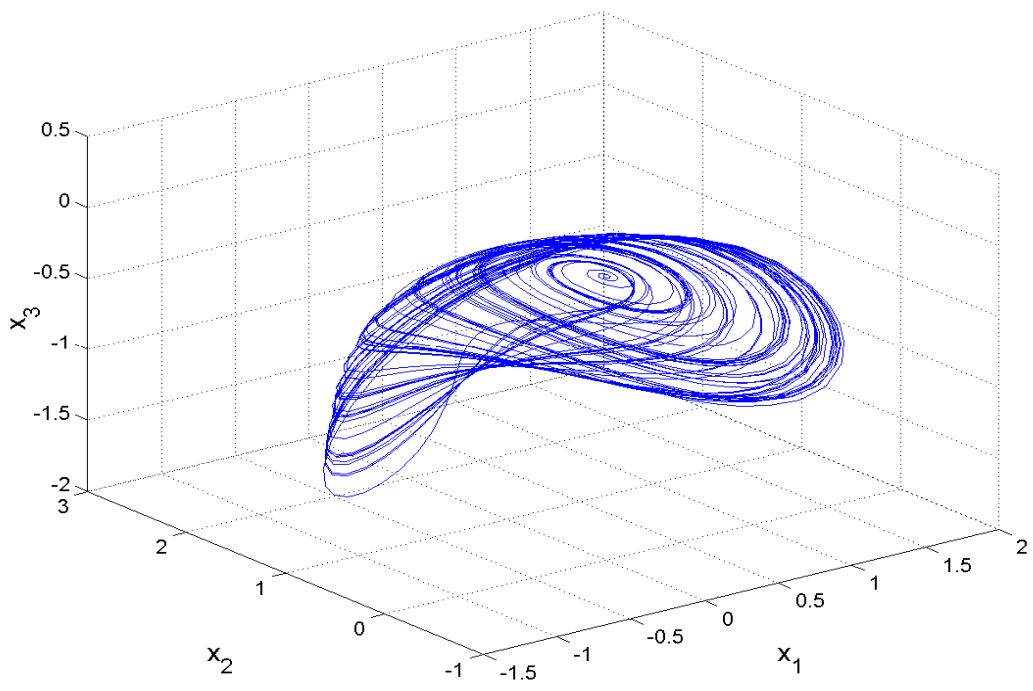

Figure 1. State Orbits of the Rössler Prototype-4 Chaotic System

When the parameter values are taken as in (2), the system (1) is chaotic and the system linearization matrix at the equilibrium point $E_{0}=(0,0,0)$ is given by

$$
A=\left[\begin{array}{ccc}
0 & -1 & -1 \\
1 & 0 & 0 \\
0 & 0.5 & -0.5
\end{array}\right]
$$

which has the eigenvalues

$$
\lambda_{1}=0.1519+1.1050 i, \lambda_{2}=0.1519-1.1050 i \text { and } \lambda_{3}=-0.8038 .
$$

Since $\lambda_{1}$ and $\lambda_{2}$ are eigenvalues with positive real part, it is immediate from Lyapunov stability theory [40] that the system (1) is unstable at the equilibrium point $E_{0}=(0,0,0)$.

In this section, we design adaptive control law for globally stabilizing the Rössler prototype-4 chaotic system (1) when the parameter values are unknown.

Thus, we consider the controlled Rössler prototype- 4 system given by the dynamics

$$
\begin{aligned}
& \dot{x}_{1}=-x_{2}-x_{3}+u_{1} \\
& \dot{x}_{2}=x_{1}+u_{2} \\
& \dot{x}_{3}=a\left(x_{2}-x_{2}^{2}\right)-b x_{3}+u_{3}
\end{aligned}
$$

where $u_{1}, u_{2}$ and $u_{3}$ are feedback controllers to be designed using the states and estimates of the unknown parameters of the system. 
In order to ensure that the controlled system (3) globally converges to the origin asymptotically, we consider the following adaptive control functions

$$
\begin{aligned}
& u_{1}=x_{2}+x_{3}-k_{1} x_{1} \\
& u_{2}=-x_{1}-k_{2} x_{2} \\
& u_{3}=-\hat{a}\left(x_{2}-x_{2}^{2}\right)+\hat{b} x_{3}-k_{3} x_{3}
\end{aligned}
$$

where $\hat{a}$ and $\hat{b}$ are estimates of the parameters $a$ and $b$, respectively, and $k_{i},(i=1,2,3)$ are positive constants.

Substituting the control law (4) into the chaotic Rössler prototype-4 system (3), we obtain

$$
\begin{aligned}
& \dot{x}_{1}=-k_{1} x_{1} \\
& \dot{x}_{2}=-k_{2} x_{2} \\
& \dot{x}_{3}=(a-\hat{a})\left(x_{2}-x_{2}^{2}\right)-(b-\hat{b}) x_{3}-k_{3} x_{3}
\end{aligned}
$$

Let us now define the parameter errors as

$$
e_{a}=a-\hat{a} \text { and } \quad e_{b}=b-\hat{b}
$$

Using (6), the closed-loop dynamics (5) can be written compactly as

$$
\begin{aligned}
& \dot{x}_{1}=-k_{1} x_{1} \\
& \dot{x}_{2}=-k_{2} x_{2} \\
& \dot{x}_{3}=e_{a}\left(x_{2}-x_{2}^{2}\right)-e_{b} x_{3}-k_{3} x_{3}
\end{aligned}
$$

For the derivation of the update law for adjusting the parameter estimates $\hat{a}, \hat{b}$, the Lyapunov approach is used.

Consider the quadratic Lyapunov function

$$
V\left(x_{1}, x_{2}, x_{3}, e_{a}, e_{b}\right)=\frac{1}{2}\left(x_{1}^{2}+x_{2}^{2}+x_{3}^{2}+e_{a}^{2}+e_{b}^{2}\right)
$$

which is a positive definite function on $R^{5}$.

Note also that

$$
\dot{e}_{a}=-\dot{\hat{a}} \text { and } \dot{e}_{b}=-\dot{\hat{b}}
$$

Differentiating $V$ along the trajectories of (7) and using (9), we obtain

$$
\dot{V}=-k_{1} x_{1}^{2}-k_{2} x_{2}^{2}-k_{3} x_{3}^{2}+e_{a}\left[x_{3}\left(x_{2}-x_{2}^{2}\right)-\dot{\hat{a}}\right]+e_{b}\left[-x_{3}^{2}-\dot{\hat{b}}\right]
$$

In view of Eq. (10), the estimated parameters are updated by the following law:

$$
\begin{aligned}
& \dot{\hat{a}}=x_{3}\left(x_{2}-x_{2}^{2}\right)+k_{4} e_{a} \\
& \dot{\hat{b}}=-x_{3}^{2}+k_{5} e_{b}
\end{aligned}
$$

where $k_{4}$ and $k_{5}$ are positive constants.

Substituting (11) into (10), we get 
International Journal of Advanced Information Technology (IJAIT) Vol. 1, No.5, October 2011

$$
\dot{V}=-k_{1} x_{1}^{2}-k_{2} x_{2}^{2}-k_{3} x_{3}^{2}-k_{4} e_{a}^{2}-k_{5} e_{b}^{2}
$$

which is a negative definite function on $R^{5}$.

Thus, by Lyapunov stability theory [40], we obtain the following result.

Theorem 1. The Rössler prototype-4 chaotic system (3) with unknown parameters is globally and exponentially stabilized for all initial conditions $x(0) \in R^{3}$ by the adaptive control law (4), where the update law for the parameters is given by (11) and $k_{i},(i=1, \ldots, 5)$ are positive constants.

\subsection{Numerical Results}

For the numerical simulations, the fourth order Runge-Kutta method is used to solve the chaotic system (3) with the adaptive control law (4) and the parameter update law (11).

The parameters of the Rössler prototype-4 chaotic system are selected as $a=b=0.5$.

For the adaptive and update laws, we take $k_{i}=4, \quad(i=1,2, \ldots, 5)$.

Suppose that the initial values of the estimated parameters are $\hat{a}(0)=4$ and $\hat{b}(0)=9$.

The initial values of the Rössler prototype-4 system are taken as $x(0)=(8,9,12)$.

When the adaptive control law (4) and the parameter update law (11) are used, the controlled Rössler prototype- 4 system converges to the equilibrium $E_{0}=(0,0,0)$ exponentially as shown in Figure 2. The parameter estimates $\hat{a}(t)$ and $\hat{b}(t)$ are shown in Figure 3 .
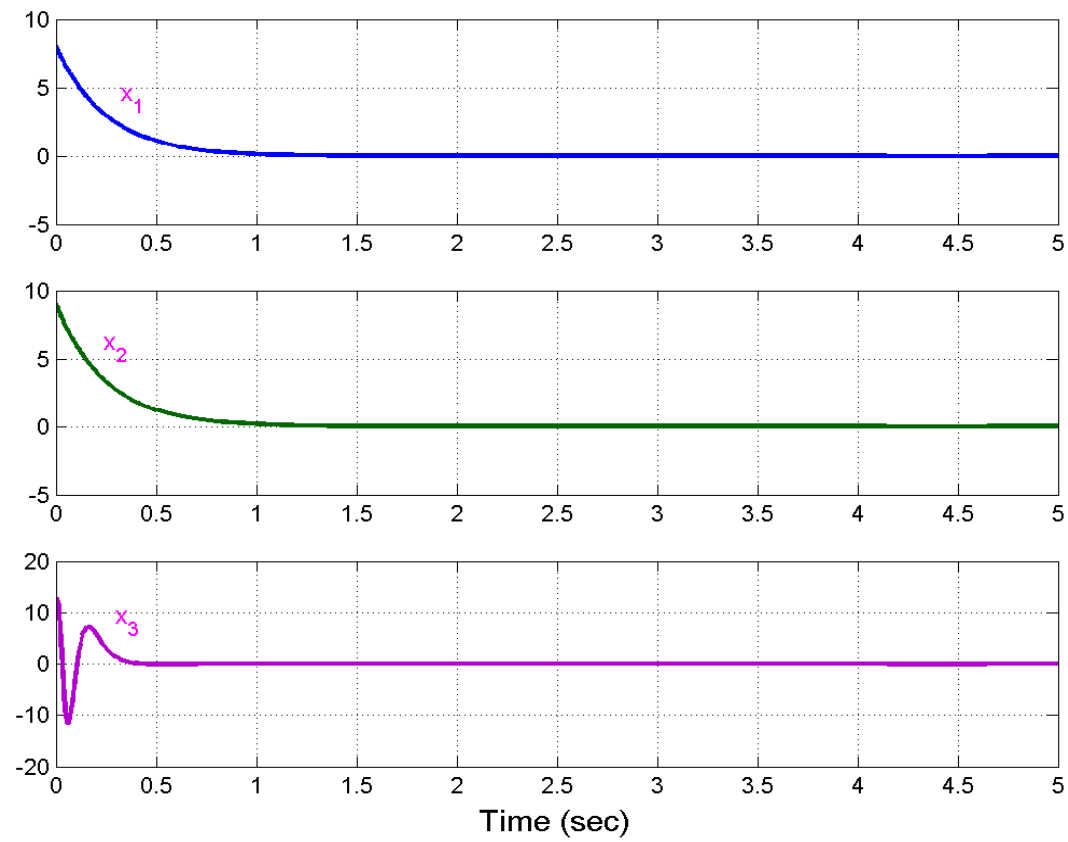

Figure 2. Time Responses of the Controlled Rössler Prototype-4 System 


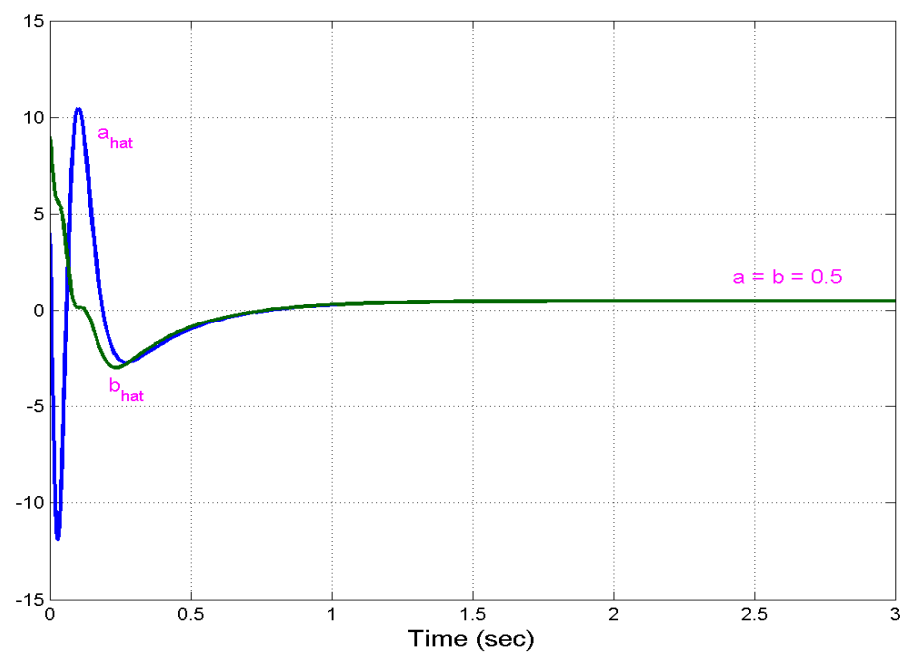

Figure 3. Parameter Estimates $\hat{a}(t), \hat{b}(t)$

\section{Adaptive Synchronization of Identical Rössler Prototype-4 CHAOTIC SYSTEMS}

\subsection{Theoretical Results}

In this section, we discuss the adaptive synchronization of identical Rössler prototype-4 chaotic systems (Rössler, 1979) with unknown parameters.

As the master system, we consider the Rössler prototype-4 dynamics described by

$$
\begin{aligned}
& \dot{x}_{1}=-x_{2}-x_{3} \\
& \dot{x}_{2}=x_{1} \\
& \dot{x}_{3}=a\left(x_{2}-x_{2}^{2}\right)-b x_{3}
\end{aligned}
$$

where $x_{i},(i=1,2,3)$ are the state variables and $a, b$ are unknown system parameters.

The system (13) is chaotic when the parameter values are taken as $a=0.5$ and $b=0.5$.

As the slave system, we consider the controlled Rössler prototype- 4 dynamics described by

$$
\begin{aligned}
& \dot{y}_{1}=-y_{2}-y_{3}+u_{1} \\
& \dot{y}_{2}=y_{1}+u_{2} \\
& \dot{y}_{3}=a\left(y_{2}-y_{2}^{2}\right)-b y_{3}+u_{3}
\end{aligned}
$$

where $y_{i},(i=1,2,3)$ are the state variables and $u_{i},(i=1,2,3)$ are the nonlinear controllers to be designed.

The synchronization error is defined by 


$$
\begin{aligned}
& e_{1}=y_{1}-x_{1} \\
& e_{2}=y_{2}-x_{2} \\
& e_{3}=y_{3}-x_{3}
\end{aligned}
$$

Then the error dynamics is obtained as

$$
\begin{aligned}
& \dot{e}_{1}=-e_{2}-e_{3}+u_{1} \\
& \dot{e}_{2}=e_{1}+u_{2} \\
& \dot{e}_{3}=a\left(e_{2}-y_{2}^{2}+x_{2}^{2}\right)-b e_{3}+u_{3}
\end{aligned}
$$

Let us now define the adaptive control functions $u_{1}(t), u_{2}(t), u_{3}(t)$ as

$$
\begin{aligned}
& u_{1}=e_{2}+e_{3}-k_{1} e_{1} \\
& u_{2}=-e_{1}-k_{2} e_{2} \\
& u_{3}=-\hat{a}\left(e_{2}-y_{2}^{2}+x_{2}^{2}\right)+\hat{b} e_{3}-k_{3} e_{3}
\end{aligned}
$$

where $\hat{a}$ and $\hat{b}$ are estimates of the parameters $a$ and $b$, respectively, and $k_{i},(i=1,2,3)$ are positive constants.

Substituting the control law (17) into (16), we obtain the error dynamics as

$$
\begin{aligned}
& \dot{e}_{1}=-k_{1} e_{1} \\
& \dot{e}_{2}=-k_{2} e_{2} \\
& \dot{e}_{3}=(a-\hat{a})\left(e_{2}-y_{2}^{2}+x_{2}^{2}\right)-(b-\hat{b}) e_{3}-k_{3} e_{3}
\end{aligned}
$$

Let us now define the parameter errors as

$$
e_{a}=a-\hat{a} \text { and } e_{b}=b-\hat{b} \text {. }
$$

Substituting (19) into (18), the error dynamics simplifies to

$$
\begin{aligned}
& \dot{e}_{1}=-k_{1} e_{1} \\
& \dot{e}_{2}=-k_{2} e_{2} \\
& \dot{e}_{3}=e_{a}\left(e_{2}-y_{2}^{2}+x_{2}^{2}\right)-e_{b} e_{3}-k_{3} e_{3}
\end{aligned}
$$

For the derivation of the update law for adjusting the estimates of the parameters, the Lyapunov approach is used.

Consider the quadratic Lyapunov function

$$
V\left(e_{1}, e_{2}, e_{3}, e_{a}, e_{b}\right)=\frac{1}{2}\left(e_{1}^{2}+e_{2}^{2}+e_{3}^{2}+e_{a}^{2}+e_{b}^{2}\right),
$$

which is a positive definite function on $R^{5}$.

Note also that

$$
\dot{e}_{a}=-\dot{\hat{a}} \text { and } \dot{e}_{b}=-\dot{\hat{b}}
$$

Differentiating $V$ along the trajectories of (20) and using (22), we obtain 


$$
\dot{V}=-k_{1} e_{1}^{2}-k_{2} e_{2}^{2}-k_{3} e_{3}^{2}+e_{a}\left[e_{3}\left(e_{2}-y_{2}^{2}+x_{2}^{2}\right)-\dot{\hat{a}}\right]+e_{b}\left[-e_{3}^{2}-\dot{\hat{b}}\right]
$$

In view of Eq. (23), the estimated parameters are updated by the following law:

$$
\begin{aligned}
& \dot{\hat{a}}=e_{3}\left(e_{2}-y_{2}^{2}+x_{2}^{2}\right)+k_{4} e_{a} \\
& \dot{\hat{b}}=-e_{3}^{2}+k_{5} e_{b}
\end{aligned}
$$

where $k_{4}$ and $k_{5}$ are positive constants.

Substituting (24) into (23), we get

$$
\dot{V}=-k_{1} e_{1}^{2}-k_{2} e_{2}^{2}-k_{3} e_{3}^{2}-k_{4} e_{a}^{2}-k_{5} e_{b}^{2},
$$

which is a negative definite function on $R^{5}$.

Thus, by Lyapunov stability theory [40], it is immediate that the synchronization error and the parameter error decay to zero exponentially with time for all initial conditions.

Hence, we have proved the following result.

Theorem 2. The identical Rössler prototype-4 chaotic systems (13) and (14) with unknown parameters are globally and exponentially synchronized for all initial conditions by the adaptive control law (17), where the update law for parameters is given by (24) and $k_{i},(i=1, \ldots, 5)$ are positive constants.

\subsection{Numerical Results}

For the numerical simulations, the fourth order Runge-Kutta method is used to solve the two systems of differential equations (13) and (14) with the adaptive control law (17) and the parameter update law (24).

The parameter values of the Rössler prototype- 4 chaotic systems are taken as

$$
a=0.5 \text { and } b=0.5
$$

We take the positive constants $k_{i},(i=1, \ldots, 5)$ as

$$
k_{i}=4 \text { for } i=1,2, \ldots, 5 .
$$

Suppose that the initial values of the estimated parameters are

$$
\hat{a}(0)=8 \text { and } \hat{b}(0)=5 .
$$

We take the initial values of the master system (13) as

$$
x_{1}(0)=4, \quad x_{2}(0)=3, \quad x_{3}(0)=8 .
$$

We take the initial values of the slave system (14) as

$$
y_{1}(0)=1, \quad y_{2}(0)=9, \quad y_{3}(0)=2 .
$$

Figure 4 shows the adaptive chaos synchronization of the identical Rössler prototype- 4 chaotic systems (13) and (14).

Figure 5 shows that the estimated values of the parameters $\hat{a}$ and $\hat{d}$ converge to the system parameters $a=0.5$ and $b=0.5$. 

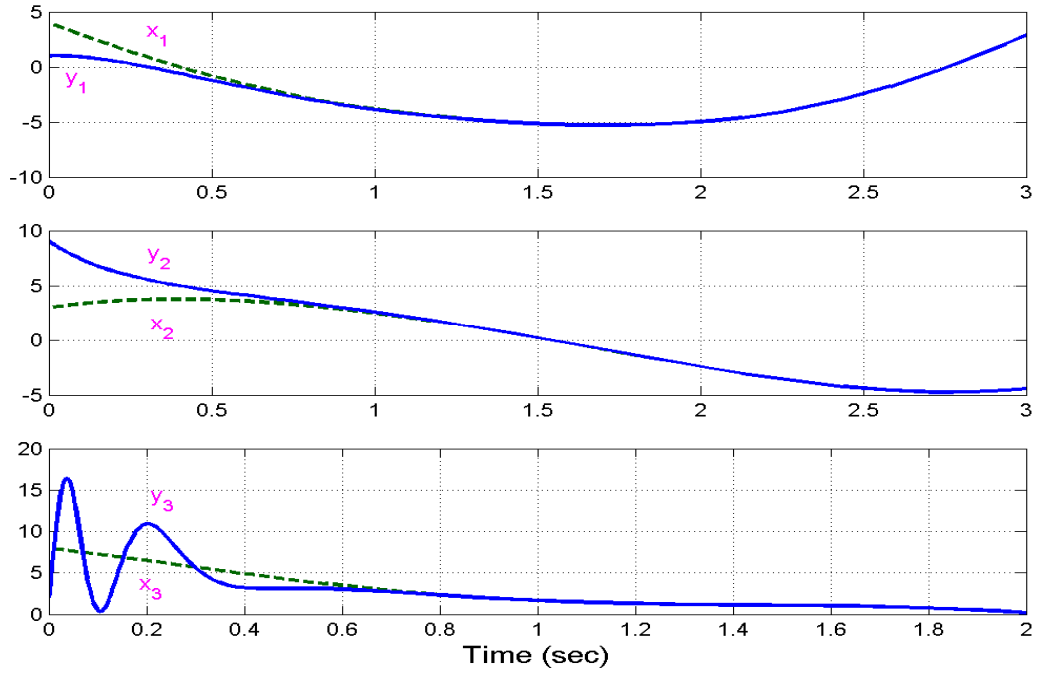

Figure 4. Adaptive Synchronization of Identical Rössler Prototype-4 Chaotic Systems

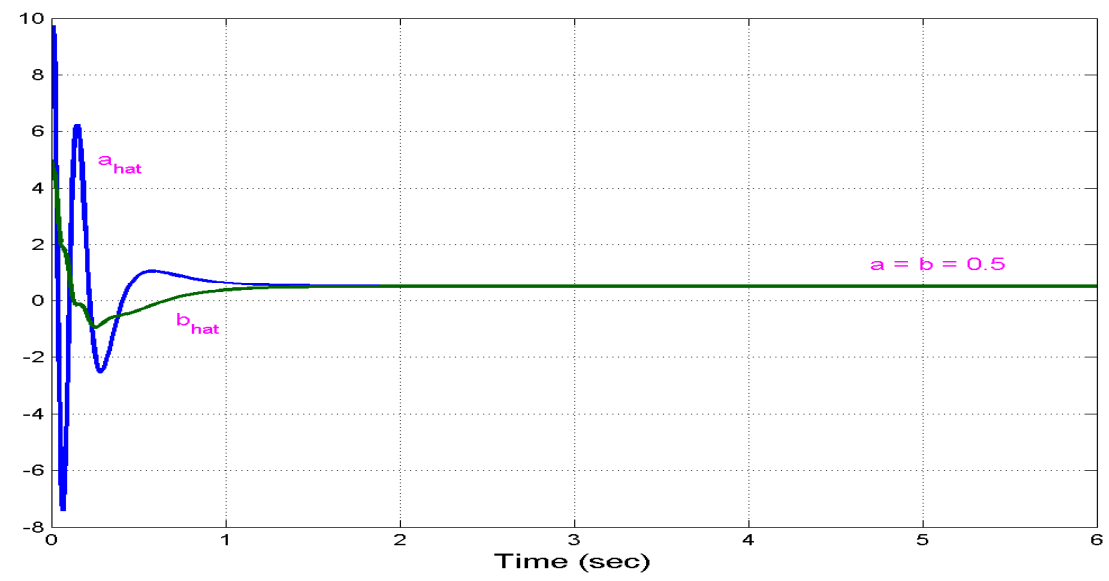

Figure 5. Parameter Estimates $\hat{a}(t), \hat{b}(t)$

\section{Conclusions}

In this paper, we applied adaptive control theory for the stabilization and synchronization of the Rössler prototype-4 chaotic system (Rössler, 1979) with unknown system parameters. First, we designed adaptive control laws to stabilize the Rössler prototype-4 chaotic system to its unstable equilibrium point at the origin based on the adaptive control theory and Lyapunov stability theory. Then we derived adaptive synchronization scheme and update law for the estimation of system parameters for identical Rössler prototype- 4 chaotic systems with unknown parameters. Our synchronization schemes were established using adaptive control theory and Lyapunov stability theory. Since the Lyapunov exponents are not required for these calculations, the proposed adaptive control method is very effective and convenient to achieve chaos control and synchronization of the Rössler prototype-4 chaotic system. Numerical simulations are shown to demonstrate the effectiveness of the proposed adaptive stabilization and synchronization schemes for the Rössler prototype-4 chaotic system. 
International Journal of Advanced Information Technology (IJAIT) Vol. 1, No.5, October 2011

\section{REFERENCES}

[1] Alligood, K.T., Sauer, T. \& Yorke, J.A. (1997) Chaos: An Introduction to Dynamical Systems, Springer, New York.

[2] Lorenz, E.N. (1963) "Deterministic nonperiodic flow", J. Atmos. Sci., Vol. 20, pp 130-141.

[3] Rössler, O.E. (1976) “An equation for continuous chaos”, Physics Letters A, Vol. 57, No. 5, pp 397 398.

[4] Chen, G. \& Ueta, T. (1999) "Yet another chaotic attractor", Internat. J. Bifurcation and Chaos, Vol. 9, No. 7, pp 1465-1466.

[5] Lü, J. \& Chen, G. (2002) “A new chaotic attractor coined”, Internat. J. Bifurcation and Chaos, Vol. 12, No. 3, pp 659-661.

[6] Liu, C., Liu, T., Liu, L. \& Liu, K. (2004) “A new chaotic attractor", Chaos, Solitons \& Fractals, Vol. 22, No. 5, pp 1031-1038.

[7] Ge, S.S., Wang, C. \& Lee, T.H. (2000) "Adaptive backstepping control of a class of chaotic systems," Internat. J. Bifur. Chaos, Vol. 10, pp 1149-1156.

[8] Wang, X., Tian, L. \& Yu, L. (2006) "Adaptive control and slow manifold analysis of a new chaotic system,” Internat. J. Nonlinear Science, Vol. 21, pp 43-49.

[9] Sun, M., Tian, L., Jiang, S. \& Xun, J. (2007) "Feedback control and adaptive control of the energy resource chaotic system," Chaos, Solitons \& Fractals, Vol. 32, pp 168-180.

[10] Astrom, K.J. \& Wittenmark, B. (1994) Adaptive Control, $2^{\text {nd }}$ edition, Prentice Hall, New Jersey, U.S.A.

[11] Pecora, L.M. \& Carroll, T.L. (1990) "Synchronization in chaotic systems", Phys. Rev. Lett., Vol. 64, pp 821-824.

[12] Lakshmanan, M. \& Murali, K. (1996) Nonlinear Oscillators: Controlling and Synchronization, World Scientific, Singapore.

[13] Han, S.K., Kerrer, C. \& Kuramoto, Y. (1995) "Dephasing and bursting in coupled neural oscillators", Phys. Rev. Lett., Vol. 75, pp 3190-3193.

[14] Blasius, B., Huppert, A. \& Stone, L. (1999) "Complex dynamics and phase synchronization in spatially extended ecological system", Nature, Vol. 399, pp 354-359.

[15] Feki, M. (2003) "An adaptive chaos synchronization scheme applied to secure communication", Chaos, Solitons and Fractals, Vol. 18, pp 141-148.

[16] Murali, K. \& Lakshmanan, M. (1998) "Secure communication using a compound signal from generalized synchronizable chaotic systems", Phys. Rev. Lett. A, Vol. 241, pp 303-310.

[17] Boccaletti, S., Farini, A. \& Arecchi, F.T. (1994) "Adaptive synchronization of chaos for secure communication,” Physical Review E., Vol. 55, No. 5, pp 4979-4981.

[18] Ott, E., Grebogi, C. \& Yorke, J.A. (1990) "Controlling chaos", Phys. Rev. Lett., Vol. 64, pp 11961199.

[19] Ho, M.C. \& Hung, Y.C. (2002) "Synchronization of two different chaotic systems using generalized active network," Physics Letters A, Vol. 301, pp 424-428.

[20] Huang, L., Feng, R. \& Wang, M. (2005) "Synchronization of chaotic systems via nonlinear control," Physical Letters A, Vol. 320, pp 271-275.

[21] Chen, H.K. (2005) "Global chaos synchronization of new chaotic systems via nonlinear control," Chaos, Solitons \& Fractals, Vol. 23, pp 1245-1251.

[22] Sundarapandian, V. \& Suresh, R. (2011) "Global chaos synchronization of hyperchaotic Qi and Jia systems by nonlinear control," International Journal of Distributed and Parallel Systems, Vol. 2, No. 2, pp 83-94.

[23] Sundarapandian, V. (2011) "Hybrid chaos synchronization of hyperchaotic Liu and hyperchaotic Chen systems by active nonlinear control," International Journal of Computer Science, Engineering and Information Technology, Vol. 1, No. 2, pp 1-14.

[24] Sundarapandian, V. \& Karthikeyan, R. (2011) "Active controller design for global chaos antisynchronization of Li and Tigan chaotic systems," International Journal of Computer Science and Information Technology, Vol. 3, No. 4, pp 255-268.

[25] Lu, J., Wu, X., Han, X. \& Lü, J. (2004) "Adaptive feedback synchronization of a unified chaotic system," Physics Letters A, Vol. 329, pp 327-333.

[26] Sundarapandian, V. (2011) "Adaptive control and synchronization of hyperchaotic Liu system," International Journal of Computer Science, Engineering and Information Technology, Vol. 1, No. 2, pp 29-40. 
International Journal of Advanced Information Technology (IJAIT) Vol. 1, No.5, October 2011

[27] Sundarapandian, V. (2011) "Adaptive control and synchronization of hyperchaotic Newton-Leipnik system,” International Journal of Advanced Information Technology, Vol. 1, No. 3, pp 22-33.

[28] Sundarapandian, V. (2011) “Adaptive synchronization of hyperchaotic Lorenz and hyperchaotic Lü systems," International Journal of Instrumentation and Control Systems, Vol. 1, No. 1, pp. 1-18.

[29] Sundarapandian, V. (2011) "Adaptive control and synchronization of hyperchaotic Cai system," International Journal of Control Theory and Computer Modeling, Vol. 1, No. 1, pp 1-13.

[30] Sundarapandian, V. (2011) "Adaptive control and synchronization of the uncertain Sprott J system," International Journal of Mathematics and Scientific Computing, Vol. 1, No. 1, pp 14-18.

[31] Yang, T. \& Chua, L.O. (1999) "Control of chaos using sampled-data feedback control", Internat. J. Bifurcat. Chaos, Vol. 9, pp 215-219.

[32] Park, J.H. \& Kwon, O.M. (2003) “A novel criterion for delayed feedback control of time-delay chaotic systems", Chaos, Solitons and Fractals, Vol. 17, pp 709-716.

[33] Yu, Y.G. \& Zhang, S.C. (2006) "Adaptive backstepping synchronization of uncertain chaotic systems", Chaos, Solitons and Fractals, Vol. 27, pp 1369-1375.

[34] Konishi, K.., Hirai, M. \& Kokame, H. (1998) "Sliding mode control for a class of chaotic systems", Phys. Lett. A, Vol. 245, pp 511-517.

[35] Sundarapandian, V. and S. Sivaperumal (2011) "Anti-synchronization of hyperchaotic Lorenz systems by sliding mode control," International Journal on Computer Science and Engineering, Vol. 3, No. 6, pp 2438-2449.

[36] Sundarapandian, V. (2011) "Sliding mode controller design for synchronization of Shimizu-Morioka chaotic systems," International Journal of Information Sciences and Techniques, Vol. 1, No. 1, pp 20-29.

[37] Sundarapandian, V. (2011) "Global chaos synchronization of four-wing chaotic systems by sliding mode control," International Journal of Control Theory and Computer Modeling, Vol. 1, No. 1, pp 15-31.

[38] Sundarapandian, V. (2011) "Global chaos synchronization of hyperchaotic Newton-Leipnik systems by sliding mode control," International Journal of Information Technology, Convergence and Services, Vol. 1, No. 4, pp 34-43.

[39] Rössler, O.E. (1979) "Continuous chaos - four prototype equations," Annals of the New York Academy of Sciences, Vol. 316, pp 376-392.

[40] Hahn, W. (1967) The Stability of Motion, Springer, New York, U.S.A.

\section{Author}

Dr. V. Sundarapandian is a Professor (Systems and Control Engineering), Research and Development Centre at Vel Tech Dr. RR \& Dr. SR Technical University, Chennai, India. His current research areas are: Linear and Nonlinear Control Systems, Chaos Theory, Dynamical Systems and Stability Theory, Soft Computing, Operations Research, Numerical Analysis and Scientific Computing, Population Biology, etc. He has published over 180 research articles in international journals and two text-books with Prentice-Hall

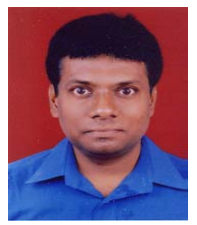
of India, New Delhi, India. He has published over 50 papers in International Conferences and 100 papers in National Conferences. He is the Editor-in-Chief of the AIRCC control journals - International Journal of Instrumentation and Control Systems, International Journal of Control Theory and Computer Modeling, and International Journal of Information Technology, Control and Automation. He is an Associate Editor of the journals - International Journal of Information Sciences and Techniques, International Journal of Control Theory and Applications, International Journal of Computer Information Systems, International Journal of Advances in Science and Technology. He has delivered several Key Note Lectures on Control Systems, Chaos Theory, Scientific Computing, MATLAB, SCILAB, etc. 